\title{
Comparison of Predictive Factors for Postoperative Incontinence of Holmium Laser Enucleation of the Prostate by the Surgeons' Experience During Learning Curve
}

\author{
Katsumi Shigemura, Kazushi Tanaka, Fukashi Yamamichi, Koji Chiba, Masato Fujisawa \\ Division of Urology, Department of Organ Therapeutics, Faculty of Medicine, Kobe University Graduate School of Medicine, Kobe, Japan
}

Purpose: To detect predictive factors for postoperative incontinence following holmium laser enucleation of the prostate (HoLEP) according to surgeon experience (beginner or experienced) and preoperative clinical data.

Methods: Of 224 patients, a total of 203 with available data on incontinence were investigated. The potential predictive factors for post-HoLEP incontinence included clinical factors, such as patient age, and preoperative urodynamic study results, including detrusor overactivity (DO). We also classified the surgeons performing the procedure according to their HoLEP experience: beginner ( $<21$ cases) and experienced ( $\geq 21$ cases).

Results: Our statistical data showed DO was a significant predictive factor at the super-short period (the next day of catheter removal: odds ratio $[\mathrm{OR}], 3.375 ; \mathrm{P}=0.000$ ). Additionally, patient age, surgeon mentorship (inverse correlation), and prostate volume were significant predictive factors at the 1-month interval after $\operatorname{HoLEP}(\mathrm{OR}, 1.072 ; \mathrm{P}=0.004 ; \mathrm{OR}, 0.251 ; \mathrm{P}=0.002$; and $\mathrm{OR}, 1.008 ; \mathrm{P}=0.049$, respectively). With regards to surgeon experience, $\mathrm{DO}$ and preoperative International Prostate Symptom Score (inverse) at the super-short period, and patient age and mentorship (inverse correlation) at the 1-month interval after HoLEP (OR, 3.952; $\mathrm{P}=0.002 ; \mathrm{OR}, 1.084 ; \mathrm{P}=0.015$; and $\mathrm{OR}, 1.084 ; \mathrm{P}=0.015 ; \mathrm{OR}, 0.358 ; \mathrm{P}=0.003$, respectively) were significant predictive factors for beginners, and first desire to void (FDV) at 1 month after $\operatorname{HoLEP}(\mathrm{OR}, 1.009 ; \mathrm{P}=0.012)$ was a significant predictive factor for experienced surgeons in multivariate analysis.

Conclusions: Preoperative DO, IPSS, patient age, and surgeon mentorship were significant predictive factors of postoperative patient incontinence for beginner surgeons, while FDV was a significant predictive factors for experienced surgeons. These findings should be taken into account by surgeons performing HoLEP to maximize the patient's quality of life with regards to urinary continence.

Keywords: Urinary Incontinence; Holmium-YAG Lasers; Transurethral Resection of Prostate; Learning curve; Urodynamics

- Conflict of Interest: No potential conflict of interest relevant to this article was reported.

\section{INTRODUCTION}

Holmium laser enucleation of the prostate (HoLEP) is performed, more routinely than before, for the surgical management of benign prostate hyperplasia (BPH) as it is becoming a substitute for transurethral resection of the prostate (TURP), especially in specific groups of patients with enlarged prostates [1]. HoLEP is considered a more accessible technique, but does involve a particularly steep learning curve in comparison with other surgical modalities for BPH [2]. Even though HoLEP is now widespread and offers good patient outcomes for lower urinary tract symptoms (LUTS), with less blood loss during surgery and minimal
Corresponding author: Katsumi Shigemura (iD http://orcid.org/0000-0002-5695-2794 Department of Urology, Kobe University Graduate School of Medicine, 7-5-1 Kusunoki-Cho, Chuo-Ku, Kobe 650-0017, Japan

E-mail: yutoshunta@hotmail.co.jp / Tel: +81-78-382-6155 / Fax: +81-78-382-6169

Submitted: July 24, 2015 / Accepted after revision: August 19, 2015 
influence on the prostate capsule compared to TURP and other modalities [3], postoperative urinary incontinence remains a representative complication of HoLEP and can have a negative influence on the patient's quality of life (QoL) [4]. This adverse event is of particular concern in the cases encountered by surgeons during the learning curve. Montorsi et al. [5] reported that urinary incontinence was more frequent after HoLEP than after other modalities, including TURP. Several studies have since sought to identify predictive factors for post-HoLEP incontinence [6]; however, most of these studies have chosen subjective evaluations. For instance, they have considered symptom scores, such as the International Prostate Symptom Score (IPSS), or surgery-related factors, such as surgical time and surgeon experience or enucleation efficiency, as potential predictive factors. More importantly, this type of study has usually been conducted within a high-volume center by experienced surgeons and, as such, information on patient outcomes associated with surgeon experience, particularly for beginner surgeons, is generally lacking.

Regarding the influence of surgeon experience on post-HoLEP incontinence in patients, Kim et al. [7] concluded that the surgeon's skills with the HoLEP procedure might plateau after approximately 25 cases. The overall occurrence of postoperative incontinence has been reported to be approximately $16.2 \%$ [4]. Kwon et al. [8] later showed that involuntary detrusor contractions in urodynamic studies (UDS) were significantly improved after surgical management with HoLEP.

In this study, we evaluated potential predictive factors for postoperative incontinence following HoLEP, focusing on surgeon experience (beginner or experienced) in combination with preoperative UDS data.

\section{MATERIALS AND METHODS}

\section{Patients and Surgeons}

Of all the procedures performed $(n=224)$ at Kobe University Hospital from 2006 to 2014, a total of 203 patients, for whom data on urinary incontinence was available, were included in this study. Urinary incontinence was defined as involuntary leakage of urine, in accordance with the recommendations of the International Continence Society [9], that required the use of pads. Incontinence was evaluated at the super-short period (the day after catheter removal), and at 1 and 3 months after HoLEP. We defined a mentor (mentorship) as a surgeon who had experience with 50 or more cases of HoLEP and included only one mentor in this study. This is a retrospective study and all study-related procedures were performed in accordance with the ethical standards laid down in the 1964 Declaration of Helsinki and its later amendments.

\section{Urodynamic Examination}

Urodynamic examination, including free uroflowmetry, filling cystometry, and pressure flow studies was performed before HoLEP [10]. We obtained informed consent by way of patient signatures for UDS. Briefly, 5-Fr feeding tubes were inserted transurethrally for bladder filling and for measuring intravesical pressure during cystometry, and an 8-Fr tube was inserted into the rectum to measure rectal pressure. The parameters investigated included maximum cystometric capacity, bladder volume at the time of the first involuntary detrusor contraction, detrusor pressure at the time of the highest involuntary detrusor contraction, and bladder compliance. The primary outcome parameters derived from the UDS results were: maximal voiding pressure (MVP), compliance, detrusor overactivity (DO), Schafer classification, volume of first desire to void (FDV), maximum cystometric capacity (MCC), and residual volume (RV) [10,11].

\section{Holmium Laser Enucleation of the Prostate}

Patients who underwent HoLEP with full UDS data were enrolled in this study. Patients with LUTS owing to BPH and who were also suspected of having prostate cancer underwent prostate biopsies first, and only patients without cancer were recommended for HoLEP. The surgical procedures carried out were as those described previously [12]. The laser setting was 72-100 W (1.8-2.5 J and 30-40 Hz). The HoLEP equipment included a high power 100-W Ho:YAG laser (VersaPulse Select; Lumenis Inc., Yokneam, Israel), a 550- $\mu \mathrm{m}$ fiber (SlimLine 550; Lumenis Inc.), a modified 26-Fr Storz continuous-flow resectoscope with a working element for stabilizing the laser fiber, a Storz rigid nephroscope, and a tissue morcellator (VersaCut system; Lumenis Inc.). Catheter indwelling time after HoLEP was 1-3 days as a rule. We herein refer to mentorship as the surgery with help by mentor as mentioned above [13].

\section{Predictive Factors for Postoperative Incontinence}

We investigated the predictive factors for postoperative incontinence following HoLEP, including potential factors, such as patient age, preoperative IPSS/QoL, the surgeon's experience (beginners: 20 cases or less; experienced: 21 cases or more), the presence of a mentor at the surgery (mentorship), and prostate size, as well as UDS factors, including MVP, compliance, DO, 
Schafer classification, FDV, MCC, and RV.

\section{Statistical Analyses}

For statistical analyses, univariate and multivariate tests were performed using StatView 5.0 software (Abacus Concepts Inc., Berkley, CA, USA). Forward stepwise logistic regression analy-

Table 1. Patients' characteristics $(n=203)$

\begin{tabular}{lc}
\hline Characteristic & Value \\
\hline Age (yr), median (range) & $71(34-90)$ \\
Prostate volume (mL) & $76.1 \pm 41.1$ \\
Resected prostate weight (g) & $52.9 \pm 35.3$ \\
Surgical time (min) & $172 \pm 63.4$ \\
Enucleation time (min) & $113 \pm 45.3$ \\
Morcellation time (min) & $29.6 \pm 28.4$ \\
Surgeons, $n$ (\%) & $84(41.4)$ \\
$\quad$ Performed by experienced & $119(58.6)$ \\
Performed by beginners & $16(7.88)$ \\
Prostate cancer, $\mathrm{n}(\%)$ &
\end{tabular}

Values are presented as mean \pm standard deviation unless otherwise indicated. sis was conducted to determine the association between various parameters and postoperative urinary incontinence status. Statistical significance was established at the level of $\mathrm{P}<0.05$.

\section{RESULTS}

\section{Patient and Surgeons}

Patient characteristics are shown in Table 1. Briefly, the median patient age was 71 years (range, 34-90 years). Resected prostate weight was $52.9 \pm 35.3 \mathrm{~g}$ (Table 1). Prostate cancer was detected in 16 cases (7.88\%). We included 11 surgeons in this study and 10 beginner surgeons performed 119 cases indicated for HoLEP. The number of patients with incontinence at the super-short period, and at 1 and 3, and 6 months after HoLEP for cases treated by beginner surgeons was 47 (39.5\%), 35 (29.4\%), 20 (16.8\%), and $6(5.04 \%)$ (data not shown), respectively, and for cases treated by experienced surgeons was 33 (39.3\%), 32 (38.1\%), 11 (13.1\%), and 4 (4.76\%) (data not shown), respectively.

\section{Predictive Factors for Post-HoLEP Incontinence}

We investigated the predictive factors for urinary incontinence

Table 2. Predictive factors for incontinence at the next day of catheter removal after HoLEP in all surgeons

\begin{tabular}{|c|c|c|c|c|c|c|}
\hline \multirow{2}{*}{ Variable } & \multicolumn{3}{|c|}{ Univariate } & \multicolumn{3}{|c|}{ Multivariate } \\
\hline & Odds ratio & $95 \% \mathrm{CI}$ & P-value & Odds ratio & $95 \% \mathrm{CI}$ & P-value \\
\hline Age & 1.038 & $0.997-1.080$ & 0.067 & & & \\
\hline Preoperative IPSS & 0.987 & $0.944-1.031$ & 0.551 & & & \\
\hline Quality of life & 0.992 & $0.687-1.434$ & 0.968 & & & \\
\hline Operation time & 1.007 & $1.003-1.012$ & $0.002^{*}$ & 0.999 & $0.988-1.011$ & 0.894 \\
\hline Enucleation time & 1.010 & $1.003-1.017$ & $0.003^{*}$ & 1.009 & $0.995-1.023$ & 0.216 \\
\hline Morcellation time & 1.008 & $0.997-1.018$ & 0.141 & & & \\
\hline Mentorship & 0.697 & $0.367-1.323$ & 0.270 & & & \\
\hline Resected prostate weight & 1.009 & $1.001-1.017$ & $0.036^{*}$ & 0.997 & $0.972-1.022$ & 0.815 \\
\hline Prostate volume & 1.007 & $1.000-1.014$ & $0.049^{*}$ & 1.006 & $0.986-1.027$ & 0.546 \\
\hline MVP & 1.007 & $1.000-1.014$ & 0.067 & & & \\
\hline Compliance & 0.990 & $0.980-1.000$ & 0.056 & & & \\
\hline Detrusor overactivity & 3.336 & $1.826-6.095$ & $<0.000^{*}$ & 3.375 & $1.790-6.363$ & $0.000^{*}$ \\
\hline Schafer & 1.171 & $0.921-1.490$ & 0.198 & & & \\
\hline First desire to void & 0.998 & $0.994-1.002$ & 0.419 & & & \\
\hline MCC & 0.998 & $0.996-1.001$ & 0.136 & & & \\
\hline Residual volume & 1.000 & $0.997-1.002$ & 0.899 & & & \\
\hline
\end{tabular}

HoLEP, holmium laser enucleation of the prostate; CI, confidence interval; IPSS, International Prostate Symptom Score; MVP, maximum voiding pressure; $M C C$, maximum cystometric capacity.

${ }^{*} \mathrm{P}<0.05$, statistical significance. 
Table 3. Predictive factors for incontinence at 1 month after HoLEP in all surgeons

\begin{tabular}{|c|c|c|c|c|c|c|}
\hline \multirow{2}{*}{ Variable } & \multicolumn{3}{|c|}{ Univariate } & \multicolumn{3}{|c|}{ Multivariate } \\
\hline & Odds ratio & $95 \% \mathrm{CI}$ & P-value & Odds ratio & $95 \% \mathrm{CI}$ & P-value \\
\hline Age & 1.086 & $1.036-1.137$ & $0.001^{*}$ & 1.072 & $1.023-1.124$ & $0.004^{*}$ \\
\hline Preoperative IPSS & 0.975 & $0.932-1.021$ & 0.281 & & & \\
\hline Quality of life & 0.749 & $0.517-1.086$ & 0.127 & & & \\
\hline Operation time & 1.005 & $1.000-1.009$ & 0.055 & & & \\
\hline Enucleation time & 1.006 & $1.000-1.013$ & 0.062 & & & \\
\hline Morcellation time & 1.005 & $0.995-1.016$ & 0.292 & & & \\
\hline Mentorship & 0.225 & $0.095-0.534$ & $0.001^{*}$ & 0.251 & $0.104-0.609$ & $0.002^{*}$ \\
\hline Resected prostate weight & 1.008 & $1.000-1.016$ & 0.057 & & & \\
\hline Prostate volume & 1.008 & $1.001-1.015$ & $0.024^{*}$ & 1.008 & $1.000-1.015$ & $0.049^{*}$ \\
\hline MVP & 1.003 & 0.995-1.010 & 0.503 & & & \\
\hline Compliance & 0.994 & $0.985-1.004$ & 0.237 & & & \\
\hline Detrusor overactivity & 1.055 & $0.582-1.910$ & 0.861 & & & \\
\hline Schafer & 1.027 & $0.809-1.303$ & 0.829 & & & \\
\hline First desire to void & 1.003 & $0.999-1.008$ & 0.107 & & & \\
\hline MCC & 1.001 & $0.998-1.003$ & 0.637 & & & \\
\hline Residual volume & 1.001 & 0.999-1.004 & 0.316 & & & \\
\hline
\end{tabular}

HoLEP, holmium laser enucleation of the prostate; CI, confidence interval; IPSS, International Prostate Symptom Score; MVP, maximum voiding pressure; MCC, maximum cystometric capacity.

${ }^{*} \mathrm{P}<0.05$, statistical significance.

Table 4. Predictive factors for incontinence at 3 months after HoLEP in all surgeons

\begin{tabular}{|c|c|c|c|c|c|c|}
\hline \multirow{2}{*}{ Variable } & \multicolumn{3}{|c|}{ Univariate } & \multicolumn{3}{|c|}{ Multivariate } \\
\hline & Odds ratio & $95 \% \mathrm{CI}$ & P-value & Odds ratio & $95 \% \mathrm{CI}$ & P-value \\
\hline Age & 1.053 & $0.996-1.114$ & 0.071 & & & \\
\hline Preoperative IPSS & 1.007 & $0.951-1.067$ & 0.802 & & & \\
\hline Quality of life & 0.943 & $0.594-1.497$ & 0.803 & & & \\
\hline Operation time & 1.003 & $0.997-1.009$ & 0.291 & & & \\
\hline Enucleation time & 1.001 & $0.992-1.010$ & 0.849 & & & \\
\hline Morcellation time & 1.004 & $0.991-1.016$ & 0.581 & & & \\
\hline Mentorship & 0.509 & $0.184-1.406$ & 0.192 & & & \\
\hline Resected prostate weight & 1.006 & $0.996-1.016$ & 0.230 & & & \\
\hline Prostate volume & 1.006 & $0.998-1.015$ & 0.143 & & & \\
\hline MVP & 0.996 & $0.986-1.006$ & 0.407 & & & \\
\hline Compliance & 0.997 & $0.984-1.010$ & 0.681 & & & \\
\hline Detrusor overactivity & 1.328 & $0.601-2.932$ & 0.483 & & & \\
\hline Schafer & 0.852 & $0.633-1.147$ & 0.290 & & & \\
\hline First desire to void & 1.005 & $1.000-1.010$ & 0.047 & 1.004 & $0.992-1.016$ & 0.516 \\
\hline MCC & 1.001 & $0.998-1.004$ & 0.496 & & & \\
\hline Residual volume & 1.001 & $0.998-1.004$ & 0.393 & & & \\
\hline
\end{tabular}

HoLEP, holmium laser enucleation of the prostate; CI, confidence interval; IPSS, International Prostate Symptom Score; MVP, maximum voiding pressure; MCC, maximum cystometric capacity. 
Table 5. Predictive factors for incontinence at the next day of catheter removal after HoLEP in beginner surgeons

\begin{tabular}{|c|c|c|c|c|c|c|}
\hline \multirow{2}{*}{ Variable } & \multicolumn{3}{|c|}{ Univariate } & \multicolumn{3}{|c|}{ Multivariate } \\
\hline & Odds ratio & $95 \% \mathrm{CI}$ & P-value & Odds ratio & $95 \% \mathrm{CI}$ & P-value \\
\hline Age & 1.021 & $0.971-1.073$ & 0.422 & & & \\
\hline Preoperative IPSS & 0.933 & $0.879-0.990$ & $0.022^{*}$ & 0.892 & $0.828-0.962$ & $0.003^{*}$ \\
\hline Quality of life & 1.113 & $0.707-1.752$ & 0.643 & & & \\
\hline Operation time & 1.003 & $0.998-1.009$ & 0.257 & & & \\
\hline Enucleation time & 1.003 & $0.994-1.012$ & 0.490 & & & \\
\hline Morcellation time & 1.003 & $0.991-1.016$ & 0.585 & & & \\
\hline Mentorship & 0.642 & $0.305-1.350$ & 0.243 & & & \\
\hline Resected prostate weight & 1.006 & $0.995-1.018$ & 0.273 & & & \\
\hline Prostate volume & 1.005 & $0.994-1.015$ & 0.396 & & & \\
\hline MVP & 1.009 & $1.000-1.019$ & 0.055 & & & \\
\hline Compliance & 0.991 & $0.979-1.002$ & 0.118 & & & \\
\hline Detrusor overactivity & 3.561 & $1.568-8.089$ & $0.002^{*}$ & 7.069 & $2.235-22.358$ & $0.001^{*}$ \\
\hline Schafer & 1.291 & $0.922-1.807$ & 0.137 & & & \\
\hline First desire to void & 0.994 & $0.988-1.000$ & 0.044 & 0.992 & $0.980-1.004$ & 0.189 \\
\hline MCC & 0.996 & $0.992-0.999$ & 0.019 & 1.000 & 0.993-1.007 & 0.961 \\
\hline Residual volume & 0.999 & $0.995-1.003$ & 0.504 & & & \\
\hline
\end{tabular}

HoLEP, holmium laser enucleation of the prostate; CI, confidence interval; IPSS, International Prostate Symptom Score; MVP, maximum voiding pressure; MCC, maximum cystometric capacity.

${ }^{*} \mathrm{P}<0.05$, statistical significance.

Table 6. Predictive factors for incontinence at 1 month after HoLEP in beginner surgeons

\begin{tabular}{|c|c|c|c|c|c|c|}
\hline \multirow{2}{*}{ Variable } & \multicolumn{3}{|c|}{ Univariate } & \multicolumn{3}{|c|}{ Multivariate } \\
\hline & Odds ratio & $95 \% \mathrm{CI}$ & $\mathrm{P}$-value & Odds ratio & $95 \% \mathrm{CI}$ & P-value \\
\hline Age & 1.099 & $1.028-1.174$ & $0.005^{*}$ & 1.084 & $1.016-1.157$ & $0.015^{*}$ \\
\hline Preoperative IPSS & 0.961 & $0.900-1.026$ & 0.234 & & & \\
\hline Quality of life & 0.818 & $0.515-1.299$ & 0.395 & & & \\
\hline Operation time & 1.004 & $0.998-1.011$ & 0.169 & & & \\
\hline Enucleation time & 1.007 & $0.997-1.016$ & 0.172 & & & \\
\hline Morcellation time & 1.001 & $0.988-1.014$ & 0.855 & & & \\
\hline Mentorship & 0.304 & $0.127-0.730$ & $0.008^{*}$ & 0.358 & $0.145-0.883$ & $0.026^{*}$ \\
\hline Resected prostate weight & 1.004 & $0.992-1.016$ & 0.520 & & & \\
\hline Prostate volume & 1.001 & $0.990-1.013$ & 0.838 & & & \\
\hline MVP & 0.999 & 0.989-1.009 & 0.880 & & & \\
\hline Compliance & 0.995 & $0.984-1.007$ & 0.404 & & & \\
\hline Detrusor overactivity & 1.203 & $0.531-2.727$ & 0.658 & & & \\
\hline Schafer & 0.974 & $0.700-1.356$ & 0.878 & & & \\
\hline First desire to void & 1.000 & $0.994-1.006$ & 0.926 & & & \\
\hline MCC & 0.998 & $0.995-1.002$ & 0.337 & & & \\
\hline Residual volume & 1.000 & $0.996-1.005$ & 0.852 & & & \\
\hline
\end{tabular}

HoLEP, holmium laser enucleation of the prostate; CI, confidence interval; IPSS, International Prostate Symptom Score; MVP, maximum voiding pressure; MCC, maximum cystometric capacity.

${ }^{*} \mathrm{P}<0.05$, statistical significance. 
Table 7. Predictive factors for incontinence at 3 months after HoLEP in beginner surgeons

\begin{tabular}{|c|c|c|c|c|c|c|}
\hline \multirow{2}{*}{ Variable } & \multicolumn{3}{|c|}{ Univariate } & \multicolumn{3}{|c|}{ Multivariate } \\
\hline & Odds ratio & $95 \% \mathrm{CI}$ & P-value & Odds ratio & $95 \% \mathrm{CI}$ & P-value \\
\hline Age & 1.064 & $0.989-1.144$ & 0.099 & & & \\
\hline Preoperative IPSS & 0.906 & $0.828-0.991$ & $0.031^{*}$ & 0.911 & $0.834-0.996$ & $0.040^{*}$ \\
\hline Quality of life & 1.081 & $0.620-1.884$ & 0.784 & & & \\
\hline Operation time & 1.008 & $1.000-1.015$ & 0.046 & 1.005 & $0.994-1.016$ & 0.375 \\
\hline Enucleation time & 1.01 & $0.998-1.021$ & 0.097 & & & \\
\hline Morcellation time & 1.004 & $0.990-1.019$ & 0.539 & & & \\
\hline Mentorship & 0.573 & $0.210-1.559$ & 0.276 & & & \\
\hline Resected prostate weight & 1.005 & $0.991-1.019$ & 0.499 & & & \\
\hline Prostate volume & 1.002 & $0.988-1.016$ & 0.767 & & & \\
\hline MVP & 1.001 & $0.989-1.013$ & 0.859 & & & \\
\hline Compliance & 1.000 & $0.989-1.011$ & 0.981 & & & \\
\hline Detrusor overactivity & 1.071 & $0.401-2.862$ & 0.891 & & & \\
\hline Schafer & 1.090 & $0.736-1.614$ & 0.668 & & & \\
\hline First desire to void & 1.003 & $0.996-1.009$ & 0.433 & & & \\
\hline MCC & 1.002 & $0.998-1.006$ & 0.454 & & & \\
\hline Residual volume & 1.003 & $0.998-1.008$ & 0.220 & & & \\
\hline
\end{tabular}

HoLEP, holmium laser enucleation of the prostate; CI, confidence interval; IPSS, International Prostate Symptom Score; MVP, maximum voiding pressure; MCC, maximum cystometric capacity.

${ }^{*} \mathrm{P}<0.05$, statistical significance.

Table 8. Predictive factors for incontinence at the next day of catheter removal after HoLEP in experienced surgeons

\begin{tabular}{|c|c|c|c|c|c|c|}
\hline \multirow{2}{*}{ Variable } & \multicolumn{3}{|c|}{ Univariate } & \multicolumn{3}{|c|}{ Multivariate } \\
\hline & Odds ratio & $95 \%$ CI & P-value & Odds ratio & $95 \%$ CI & P-value \\
\hline Age & 1.067 & $0.999-1.139$ & 0.053 & & & \\
\hline Preoperative IPSS & 0.995 & $0.925-1.072$ & 0.905 & & & \\
\hline Quality of life & 0.775 & $0.405-1.484$ & 0.442 & & & \\
\hline Operation time & 1.016 & $1.007-1.025$ & $0.001^{*}$ & 1.004 & $0.987-1.022$ & 0.625 \\
\hline Enucleation time & 1.023 & $1.011-1.036$ & $0.000^{*}$ & 1.017 & $0.994-1.041$ & 0.152 \\
\hline Morcellation time & 1.017 & $0.999-1.036$ & 0.071 & & & \\
\hline Mentorship & 0.6 & $0.425-1.024$ & 0.979 & & & \\
\hline Resected prostate weight & 1.011 & $0.999-1.023$ & 0.062 & & & \\
\hline Prostate volume & 1.009 & $1.000-1.019$ & 0.060 & & & \\
\hline MVP & 1.003 & $0.991-1.015$ & 0.612 & & & \\
\hline Compliance & 0.988 & $0.968-1.008$ & 0.242 & & & \\
\hline Detrusor overactivity & 3.208 & $1.288-7.992$ & $0.012^{*}$ & 2.624 & $0.964-7.138$ & 0.059 \\
\hline Schafer & 1.052 & $0.743-1.487$ & 0.776 & & & \\
\hline First desire to void & 1.004 & $0.997-1.010$ & 0.236 & & & \\
\hline MCC & 1 & $0.997-1.004$ & 0.773 & & & \\
\hline Residual volume & 1 & $0.998-1.003$ & 0.747 & & & \\
\hline
\end{tabular}

HoLEP, holmium laser enucleation of the prostate; CI, confidence interval; IPSS, International Prostate Symptom Score; MVP, maximum voiding pressure; MCC, maximum cystometric capacity.

${ }^{*} \mathrm{P}<0.05$, statistical significance. 
Table 9. Predictive factors for incontinence at 1 month after HoLEP in experienced surgeons

\begin{tabular}{|c|c|c|c|c|c|c|}
\hline \multirow{2}{*}{ Variable } & \multicolumn{3}{|c|}{ Univariate } & \multicolumn{3}{|c|}{ Multivariate } \\
\hline & Odds ratio & $95 \% \mathrm{CI}$ & P-value & Odds ratio & $95 \% \mathrm{CI}$ & P-value \\
\hline Age & 0.990 & $0.930-1.053$ & 0.741 & & & \\
\hline Preoperative IPSS & 1.017 & $0.948-1.092$ & 0.632 & & & \\
\hline Quality of life & 1.173 & $0.623-2.210$ & 0.621 & & & \\
\hline Operation time & 1.000 & $0.993-1.008$ & 0.926 & & & \\
\hline Enucleation time & 1.004 & $0.994-1.014$ & 0.416 & & & \\
\hline Morcellation time & 0.992 & $0.972-1.013$ & 0.465 & & & \\
\hline Mentorship & 0.733 & $0.468-1.149$ & 0.979 & & & \\
\hline Resected prostate weight & 0.995 & $0.984-1.007$ & 0.454 & & & \\
\hline Prostate volume & 0.997 & $0.987-1.007$ & 0.528 & & & \\
\hline MVP & 1.006 & $0.994-1.019$ & 0.320 & & & \\
\hline Compliance & 1.005 & $0.987-1.023$ & 0.578 & & & \\
\hline Detrusor overactivity & 1.705 & $0.692-4.204$ & 0.246 & & & \\
\hline Schafer & 1.098 & $0.771-1.562$ & 0.605 & & & \\
\hline First desire to void & 1.009 & $1.002-1.017$ & $0.012^{*}$ & & & \\
\hline MCC & 1.002 & $0.999-1.005$ & 0.243 & & & \\
\hline Residual volume & 1.000 & $0.997-1.003$ & 0.877 & & & \\
\hline
\end{tabular}

HoLEP, holmium laser enucleation of the prostate; CI, confidence interval; IPSS, International Prostate Symptom Score; MVP, maximum voiding pressure; MCC, maximum cystometric capacity.

${ }^{*} \mathrm{P}<0.05$, statistical significance.

Table 10. Predictive factors for incontinence at 3 months after HoLEP in experienced surgeons

\begin{tabular}{|c|c|c|c|c|c|c|}
\hline \multirow{2}{*}{ Variable } & \multicolumn{3}{|c|}{ Univariate } & \multicolumn{3}{|c|}{ Multivariate } \\
\hline & Odds ratio & $95 \% \mathrm{CI}$ & P-value & Odds ratio & $95 \% \mathrm{CI}$ & P-value \\
\hline Age & 0.956 & $0.873-1.048$ & 0.335 & & & \\
\hline Preoperative IPSS & 0.977 & $0.881-1.083$ & 0.658 & & & \\
\hline Quality of life & 1.261 & $0.485-3.280$ & 0.634 & & & \\
\hline Operation time & 0.990 & $0.977-1.003$ & 0.123 & & & \\
\hline Enucleation time & 0.984 & $0.966-1.003$ & 0.096 & & & \\
\hline Morcellation time & 0.994 & $0.965-1.025$ & 0.709 & & & \\
\hline Mentorship & 0.167 & $0.088-0.316$ & 0.981 & & & \\
\hline Resected prostate weight & 0.991 & $0.971-1.011$ & 0.372 & & & \\
\hline Prostate volume & 0.994 & $0.977-1.010$ & 0.451 & & & \\
\hline MVP & 0.988 & $0.969-1.007$ & 0.216 & & & \\
\hline Compliance & 1.003 & $0.979-1.027$ & 0.795 & & & \\
\hline Detrusor overactivity & 0.968 & $0.269-3.482$ & 0.960 & & & \\
\hline Schafer & 0.718 & $0.427-1.208$ & 0.212 & & & \\
\hline First desire to void & 0.995 & $0.985-1.005$ & 0.343 & & & \\
\hline $\mathrm{MCC}$ & 0.995 & $0.989-1.002$ & 0.166 & & & \\
\hline Residual volume & 0.996 & $0.990-1.003$ & 0.300 & & & \\
\hline
\end{tabular}

HoLEP, holmium laser enucleation of the prostate; CI, confidence interval; IPSS, International Prostate Symptom Score; MVP, maximum voiding pressure; MCC, maximum cystometric capacity. 
for the super-short period, and at 1 and 3 months after HoLEP. Our statistical data are shown in Tables 2-10. Briefly, in the investigation of all surgeons, preoperative DO was a significant predictive factor at the super-short period after HoLEP (odds ratio $[\mathrm{OR}], 3.336 ; \mathrm{P}=0.000)$. Even though the following variables had comparatively lower OR patient age and prostate volume were significant predictive factors at the 1-month interval after HoLEP (OR, 1.072; P = 0.004 and OR, 1.008; P =0.049, respectively). Importantly, in the multivariate analyses, mentorship during surgery was an inverse significant predictive factor at the 1-month interval $(\mathrm{OR}, 0.251 ; \mathrm{P}=0.002)$ in all patient categories.

In the classification of surgeon experience, briefly, DO and preoperative IPSS (inverse) at the super-short period after HoLEP, and patient age and mentorship (inverse correlation) at the 1 month (OR, 0.892; $\mathrm{P}=0.003$; OR, 7.069; $\mathrm{P}=0.001$ and $\mathrm{OR}$, 1.084; $\mathrm{P}=0.015 ; \mathrm{OR}, 0.358 ; \mathrm{P}=0.026$, respectively) and preoperative IPSS (inverse) (OR, $0.911 ; \mathrm{P}=0.040)$ at the 3 months for beginner surgeons, were identified as significant predictive factors in multivariate analyses, and FDV at the 1-month interval after HoLEP (OR, 1.009; $\mathrm{P}=0.012$ ) performed by experienced surgeons, was identified as a significant predictive factor in univariate analyses. These results suggest that preoperative DO is significantly correlated with incontinence at the super-short period after HoLEP considering the high OR value; for experienced surgeons, DO tended to be a predictive factor with a high OR (2.624) although this finding was not statistically significant. Importantly, mentorship significantly contributed to patient outcome with regards to continence at 1 month following HoLEP (Tables 2-10). However, no significant predictive factors were identified in either experienced surgeon category at 3-month interval after HoLEP in experienced surgeons.

\section{DISCUSSION}

Post-HoLEP incontinence has been issued as the main adverse event [14]; however, it should be prevented because patients are generally able to maintain continence preoperatively. Lerner et al. [6] stated that the risk factors for post-HoLEP stress incontinence include the number of days between cases for surgeons. On the other hand, there is a study describing a learning curve that the postoperative transient urinary incontinence rate was higher in initial cases (No. 1-50) than in the other groups (No. 51-100 and No. 101-190) [15]. Therefore, we investigated the predictive factors for post-HoLEP incontinence from the super- short period to 3 months after surgery, with a particular focus on the surgeon's level of experience. There are some arguments regarding the clinical validity of investigating the super-short period after HoLEP [16,17]; however, we believe incontinence may damage patient QoL even during the period immediately following the procedure, and, as such, we chose to include this timeframe in our examination.

In general, post-HoLEP incontinence may be due to an imbalance between the bladder's storage functions, following release of the bladder outlet obstruction, and sphincter function after HoLEP [18]. Several studies have addressed the damage to the sphincter as a risk factor for post-HoLEP incontinence, and surgical time and the surgeon's skill level can affect the risk of the sphincter damage [19]. Shah et al. [20] found, in their 280case study, that the surgeon's experience was a significant factor for post-HoLEP incontinence, because inexperienced surgeons tended to be disoriented, and may inadvertently cause sphincter damage, or may encounter delays when dealing with the membrane at the prostate apex such that tension in the membrane would damage or affect the nearby sphincter. As to the strategies for decreasing post-HoLEP incontinence, Jeong et al. [21] stated that surgeons needed to have experience with at least 25 cases to optimize their technique, even though the learning curve for HoLEP is steep compared to other modalities, such as TURP. In addition, the presence of a mentor (mentorship) at the surgery tended to decrease surgical complications and offer better patient outcomes when compared with surgeries where no mentor was present [22]. Related to this issue, our data showed that in particular DO and mentorship (inverse correlation) were significant predictors for post-HoLEP incontinence for beginner surgeons. Moreover, FDV was a significant predictive factor for experienced surgeons, suggesting, taken together, that mentorship contributes to the prevention of post-HoLEP incontinence and the education system works well for this purpose.

Bruschini et al. [23] stated that preoperative DO or urethral sphincter insufficiency was a significant factor in urinary incontinence following surgery for BPH. Our UDS and post-HoLEP incontinence data also showed that DO was a significant factor in the super-short period after HoLEP (the next day of catheter removal) and this finding is emphasized for non-experienced surgeons.

Our data also showed that patient age was a significant predictive factor for incontinence at the 1-month interval for beginner surgeons. However, Lerner et al. [6] stated that they 
found no significant differences in patient age between those with and without incontinence at 3 months following HoLEP. Elmansy et al. [18] reported that diabetes mellitus, but not patient age, was significantly associated with a higher incidence of incontinence. This difference could be attributed to the variation in sample sizes: $n=66$ (Lerner et al. [6]) and $n=954$ (Elmansy et al. [18]) vs. $n=203$ in our study, as well as, the number of surgeons included. To our knowledge, there are no reports that have investigated the predictive factors for post-HoLEP incontinence, as classified according to the surgeons' level of experience. Our data showed that the significant predictive factors identified for post-HoLEP in each surgeon category (based on experience) are informative, even for surgeons with a variety of experience. However, we identified no significant predictive factors in the investigation at 3 months after HoLEP in experienced surgeons; therefore, a longer period of observation is necessary as well.

A number of study limitations also remain to be addressed. First, this is a retrospective audit of patient outcomes from a single-center study. Second, several UDS parameters, including urethral length and maximum urethral closure pressure, were not assessed. Third, we did not investigate severity or type, as well as, the continuation of incontinence, with objective tests, such as the pad test or bladder diary and did not include a definitive training program for the surgeons involved in the study. Fourth, we have no available data regarding what a mentor may have pointed out in detail during the surgery, or rather, what factors may render the surgical outcome different before and after 21 HoLEP cases for beginner surgeons. However, these data are from retrospective studies conducted in teaching hospitals, implying that this study could potentially be undertaken in all institutions where HoLEP is performed. As such, the above stated limitations should be overcome in future prospective studies.

In conclusion, we found that predictive factors for post-HoLEP incontinence had variations in their time course. Especially preoperative $\mathrm{DO}$ at the super-short period and patient age, surgeon mentorship, and prostate volume at 1 month after HoLEP, were the representative significant predictive factors identified during our 3-month follow-up duration. Additionally, preoperative DO and surgeon mentorship were representative significant factors for beginner surgeons, while only FDV age was a significant factor for experienced surgeons. These findings should be taken into account by surgeons, in particular beginners, to maximize the patient's QoL with regards to urinary continence.

\section{ACKNOWLEDGEMENTS}

We thank Dr. Takahiro Haraguchi for taking care of the patients.

\section{REFERENCES}

1. Elzayat EA, Habib EI, Elhilali MM. Holmium laser enucleation of the prostate: a size-independent new "gold standard". Urology 2005;66(5 Suppl):108-13.

2. Cho KJ, Kim HS, Koh JS, Han SB, Kim SH, Kim HW, et al. Preoperative factors affecting postoperative early quality of life during the learning curve of holmium laser enucleation of the prostate. Int Neurourol J 2013;17:83-9.

3. Kelly DC, Das A. Holmium laser enucleation of the prostate technique for benign prostatic hyperplasia. Can J Urol 2012;19:6131-4.

4. Cho MC, Park JH, Jeong MS, Yi JS, Ku JH, Oh SJ, et al. Predictor of de novo urinary incontinence following holmium laser enucleation of the prostate. Neurourol Urodyn 2011;30:1343-9.

5. Montorsi F, Naspro R, Salonia A, Suardi N, Briganti A, Zanoni M, et al. Holmium laser enucleation versus transurethral resection of the prostate: results from a 2-center, prospective, randomized trial in patients with obstructive benign prostatic hyperplasia. J Urol 2004;172(5 Pt 1):1926-9.

6. Lerner LB, Tyson MD, Mendoza PJ. Stress incontinence during the learning curve of holmium laser enucleation of the prostate. J Endourol 2010;24:1655-8.

7. Kim M, Lee HE, Oh SJ. Technical aspects of holmium laser enucleation of the prostate for benign prostatic hyperplasia. Korean J Urol 2013;54:570-9.

8. Kwon O, Lee HE, Bae J, Oh JK, Oh SJ. Effect of holmium laser enucleation of prostate on overactive bladder symptoms and urodynamic parameters: a prospective study. Urology 2014;83:581-5.

9. Abrams P, Cardozo L, Fall M, Griffiths D, Rosier P, Ulmsten U, et al. The standardisation of terminology of lower urinary tract function: report from the Standardisation Sub-committee of the International Continence Society. Neurourol Urodyn 2002;21:167-78.

10. Cartwright R, Hashim H, Chapple C. What was "hot" at the ICS Annual Meeting San Francisco 2009. Neurourol Urodyn 2010;29:21-7.

11. Brown DM, Wickham WE. The urethral pressure profile. Br J Urol 1969;51:211-7.

12. Haraguchi T, Takenaka A, Yamazaki T, Nakano Y, Miyake H, Tana$\mathrm{ka} \mathrm{K}$, et al. The relationship between the reproducibility of holmium laser enucleation of the prostate and prostate size over the learning curve. Prostate Cancer Prostatic Dis 2009;12:281-4. 
13. Aho T, Herrmann TR. Description of a modular mentorship programme for holmium laser enucleation of the prostate. World J Urol 2015;33:497-502.

14. Wilson LC, Gilling PJ, Williams A, Kennett KM, Frampton CM, Westenberg AM, et al. A randomised trial comparing holmium laser enucleation versus transurethral resection in the treatment of prostates larger than 40 grams: results at 2 years. Eur Urol 2006;50:569-73.

15. Du C, Jin X, Bai F, Qiu Y. Holmium laser enucleation of the prostate: the safety, efficacy, and learning experience in China. J Endourol 2008;22:1031-6.

16. Endo F, Shiga Y, Minagawa S, Iwabuchi T, Fujisaki A, Yashi M, et al. Anteroposterior dissection HoLEP: a modification to prevent transient stress urinary incontinence. Urology 2010;76:1451-5.

17. Shenoy SP, Marla PK, Suvarna R. Re: Endo et al.: Anteroposterior dissection HoLEP: a modification to prevent transient stress urinary incontinence (Urology 2010;76:1451-1456). Urology 2011;77:1510; author reply 1510-1.

18. Elmansy HM, Kotb A, Elhilali MM. Is there a way to predict stress urinary incontinence after holmium laser enucleation of the pros- tate? J Urol 2011;186:1977-81.

19. Bae J, Choo M, Park JH, Oh JK, Paick JS, Oh SJ. Holmium laser enucleation of prostate for benign prostatic hyperplasia: seoul national university hospital experience. Int Neurourol J 2011;15:2934.

20. Shah HN, Mahajan AP, Hegde SS, Bansal MB. Peri-operative complications of holmium laser enucleation of the prostate: experience in the first 280 patients, and a review of literature. BJU Int 2007; 100:94-101.

21. Jeong CW, Oh JK, Cho MC, Bae JB, Oh SJ. Enucleation ratio efficacy might be a better predictor to assess learning curve of holmium laser enucleation of the prostate. Int Braz J Urol 2012;38:362-71.

22. Hwang JC, Park SM, Lee JB. Holmium laser enucleation of the prostate for benign prostatic hyperplasia: effectiveness, safety, and overcoming of the learning curve. Korean J Urol 2010;51:619-24.

23. Bruschini H, Simonetti R, Antunes AA, Srougi M. Urinary incontinence following surgery for $\mathrm{BPH}$ : the role of aging on the incidence of bladder dysfunction. Int Braz J Urol 2011;37:380-6. 